\title{
Constraints on nonstandard neutrino interactions from the COHERENT experiment
}

\author{
Jiajun Liao* \\ Department of Physics and Astronomy, University of Hawaii-Manoa, Honolulu, HI 96822, USA \\ E-mail: liaoj@hawaii.edu

\section{Danny Marfatia} \\ Department of Physics and Astronomy, University of Hawaii-Manoa, Honolulu, HI 96822, USA \\ E-mail: dmarf8@hawaii.edu
}

\begin{abstract}
The COHERENT experiment has detected the coherent elastic neutrino-nucleus scattering for the first time, and its measurement is consistent with the standard model (SM) prediction. We present a simple calculation of the detected spectrum, and use it to put constraints on the nonstandard neutrino interactions (NSI). For NSI generated by a very light mediator, only couplings of the mediator are constrained by the COHERENT data, and large NSI are still viable. For a heavy mediator, despite the fact that constraints are severely weakened by the degeneracies between the NSI parameters, current COHERENT data can place meaningful constraints on the effective NSI parameters in Earth matter.
\end{abstract}

The 19th International Workshop on Neutrinos from Accelerators-NUFACT2017

25-30 September, 2017

Uppsala University, Uppsala, Sweden

\footnotetext{
${ }^{*}$ Speaker.
} 


\section{Introduction}

The study of neutrino oscillations has entered the precision era. The current and future neutrino oscillation experiments will reach the sensitivity to probe new physics beyond the SM, which is often studied in the framework of NSI [1]. Indications of the existence of NSI may have already been seen from the tensions of the measurements of standard oscillation parameters in the current data, such as the apparent departure from maximal $\theta_{23}$ in NOvA [2], and the discrepancy in the current measurements of $\delta m_{21}^{2}$ from the solar and reactor neutrino experiments [3]. The presence of NSI could lead to a wrong determination of the mass hierarchy, CP violation and $\theta_{23}$ octant at current and future long baseline neutrino oscillation experiments NOvA, T2K and DUNE[4], and due to the degeneracies between the NSI parameters and by the generalized mass hierarchy degeneracy, the potential of DUNE, T2HK and T2HKK to determine the mass hierarchy and CP violation is severely impaired [5]. Hence, it is important to resort to other non-oscillation experiments to constrain NSI. The matter NSI that appeared in the neutrino oscillation experiments could also be constrained by neutrino scattering experiments. The previous CHARM and NuTeV experiments measured the neutrino-nucleus scattering cross section in the deep-inelastic regime, and their constraints on NSI can be easily evaded for NSI models with mediator mass less than about $1 \mathrm{GeV}$ [6]. The coherent neutrino-nucleus scattering (CEvNS) has a moment transfer in the tens of $\mathrm{MeV}$ region and has much larger cross section than any other processes in the SM for neutrino energy up to $50 \mathrm{MeV}$ [7]. Hence, it is desirable to use CEvNS to constrain NSI models with light mediators. However, due to the tiny nuclear recoil energy involved in CEvNS, CEvNS has not been observed until recently by the COHERENT experiment [8].

\section{Calculations}

The neutrinos measured by the COHERENT experiment are provided in the Spallation Neutron Source (SNS) at Oak Ridge National Laboratory. Several neutrino detectors exist at the facility, and the first results are measured by the CSI detector, which is can detect the nuclear recoil energies down to a few $\mathrm{keV}$ [9]. The neutrinos come as a byproduct from the pion and muon decay, which are produced by very intense pulsed proton beam hitting on the mercury target. Since all the neutrinos are produced by decay at rest, they are emitted isotropically. We calculate the expected number of CEvNS events per bin for neutrino flavor $\alpha$ by the following equation

$$
N_{\alpha}^{i}=\frac{r N_{\mathrm{POT}}}{4 \pi L^{2}} \times \frac{2 m_{\mathrm{det}}}{M_{\mathrm{CSI}}} N_{A} \times \int d n_{\mathrm{PE}} f\left(n_{\mathrm{PE}}\right) \frac{d E_{r}}{d n_{\mathrm{PE}}} \int d E_{v} \phi_{\alpha}\left(E_{v}\right) \frac{d \sigma_{\alpha}}{d E_{r}}\left(E_{v}, E_{r}\right),
$$

where $r=0.08$ is the number of neutrinos per flavor that are produced for each proton on target, $N_{\text {POT }}=1.76 \times 10^{23}$ is the total number of protons delivered to the target, $L=19.3 \mathrm{~m}$ is the distance between the source and the CsI detector, $m_{\mathrm{det}}=14.6 \mathrm{~kg}$ is the detector mass, $M_{\mathrm{CsI}}$ is the molar mass of CsI, and $N_{A}$ is the Avogadro constant. We adopt a simple relation between the observed number of photoelectrons (PE) and the nuclear recoil energy as $n_{\mathrm{PE}}=1.17 \frac{E_{r}}{\mathrm{keV}}$ [8]. Also, in order to maximize the signal-to-environmental background ratio, several cuts have been applied to the data. Here we include the surviving fraction of the CEvNS signals as a function of the number of photoelectrons $f\left(n_{\mathrm{PE}}\right)$ to take account of the effects of the cuts, which is given in Fig. S9 in Ref. [8]. 
The neutrino flux $\phi_{\alpha}\left(E_{v}\right)$ at the SNS is well known. The pions produced by the proton collision on target are quickly stopped in the target. The $\pi^{-}$'s are quickly captured by the target nuclei, and the $\pi^{+}$'s decay at rest, producing a prompt component of $v_{\mu}$ with a mono-energetic energy at about $30 \mathrm{MeV}$. The muons from the pion decay travel about one tenth of a millimeter and also decay at rest, producing two delayed components of $\bar{v}_{\mu}$ and $v_{e}$. Their energy spectra are more spread out but are well-confined to be less than half the muon mass. The differential cross section for a given neutrino flavor $v_{\alpha}$ in the SM can be written as

$$
\frac{d \sigma_{\alpha}}{d E_{r}}=\frac{G_{F}^{2}}{2 \pi} Q_{\alpha}^{2} F^{2}\left(2 M E_{r}\right) M\left(2-\frac{M E_{r}}{E_{v}^{2}}\right),
$$

where $M$ is the mass of the target nucleus, $F\left(2 M E_{r}\right)$ is the nuclear form factor [10], and $Q_{\alpha}$ is the effective charge, which can be easily modified to accommodate for different NSI scenarios. The effective charge in the SM is given by $Q_{\alpha, \mathrm{SM}}=Z g_{p}^{V}+N g_{n}^{V}$, where $g_{p}^{V}=\frac{1}{2}-2 \sin ^{2} \theta_{W}$ and $g_{n}^{V}=-\frac{1}{2}$, with $Z$ and $N$ being the number of protons and neutrons in the nucleus, and $\theta_{W}$ the weak mixing angle. As can be seen from Fig. 1 in Ref. [11], our simple calculation of the spectrum is very close to the COHERENT simulations for the SM. Also, from Fig. 1 in Ref. [11], we see that the COHERENT data is in good agreement with SM predictions, and we can use it to place constraints on new physics beyond the SM such as NSI.

\section{Results and discussion}

We first consider the case that NSI are induced by a light vector mediator $Z^{\prime}$ that has purely vector universal flavor-conserving couplings $g$ to neutrinos, first generation quarks and the muon. Then the effective charge in Eq. (2.2) can be written as

$$
Q_{\alpha, \mathrm{NSI}}^{2}=\left[Z\left(g_{p}^{V}+\frac{3 g^{2}}{2 \sqrt{2} G_{F}\left(Q^{2}+M_{Z^{\prime}}^{2}\right)}\right)+N\left(g_{n}^{V}+\frac{3 g^{2}}{2 \sqrt{2} G_{F}\left(Q^{2}+M_{Z^{\prime}}^{2}\right)}\right)\right]^{2},
$$

where $M_{Z^{\prime}}$ is the mediator mass and $Q^{2}=2 M E_{r}$ is the square of the momentum transfer. The $2 \sigma$ limit in the $\left(M_{Z^{\prime}}, g\right)$ plane obtained by using the spectrum of the COHERENT data is shown in Fig. 1. We see that a light mediator that can explain the discrepancy in the anomalous magnetic moment of the muon [12] is disfavored in this model. Since the NSI contribution is proportional to $\frac{g^{2}}{2 M E_{r}+M_{Z^{\prime}}^{2}}$, the limit is only sensitive to the coupling $g$ for $M_{Z^{\prime}} \ll \sqrt{2 M E_{r}} \sim 50 \mathrm{MeV}$. Since the NSI matter effect for neutrino propagation is sensitive to $\frac{g^{2}}{M_{Z^{\prime}}^{2}}$ [13], the COHERENT constraint cannot be directly applied to the matter NSI induced by a very light mediator. For a heavy mediator with mass $M_{Z^{\prime}} \gg 50 \mathrm{MeV}$, the moment transfer is negligible and the shape of the spectra is not affected by NSI. Since the COHERENT constraint depends on the ratio $\frac{g}{M_{Z^{\prime}}}$, it can be directly placed on the matter NSI. Also, from Fig. 1 we see that there is a degenerate region that cannot be excluded by current data. After neglecting the small momentum transfer for a heavy mediator, we can explain the degenerate region by the relation,

$$
\frac{g^{2}}{M_{Z^{\prime}}^{2}}=-\frac{4 \sqrt{2}\left(Z g_{p}^{V}+N g_{n}^{V}\right)}{3(Z+N)} G_{F}
$$


which is shown as the purple line in Fig. 1. The degeneracy holds for all $E_{r}$ bins when $M_{Z^{\prime}} \gg$ $\sqrt{2 M E_{r}}$, but is broken for a very light mediator due to the modification of the spectral shapes by the momentum transfer terms in NSI.

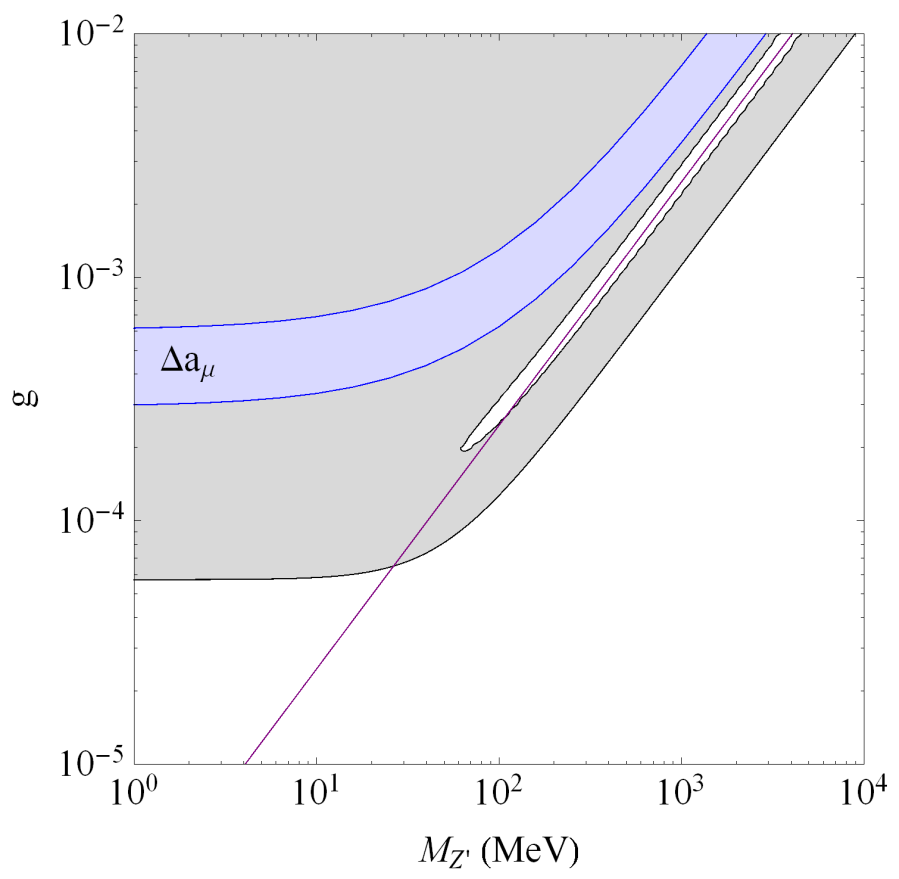

Figure 1: The $2 \sigma$ exclusion region in the $\left(M_{Z^{\prime}}, g\right)$ plane obtained by using the spectrum of the COHERENT data. We also show the $2 \sigma$ allowed region that explains the discrepancy in the anomalous magnetic moment of the muon for comparison. The purple line corresponds to the relation given by Eq. (3.2).

For a heavy mediator, since the momentum transfer is negligible, the matter NSI can be described by four-fermion contact operators as

$$
\mathscr{L}_{\mathrm{NSI}}=-\sqrt{2} G_{F} \varepsilon_{\alpha \beta}^{f V}\left[\bar{v}_{\alpha L} \gamma^{\rho} v_{\beta L}\right]\left[\bar{f} \gamma_{\rho} f\right],
$$

where $\alpha, \beta=e, \mu, \tau, f=u, d$, and we parameterize the strength of the new interaction $\varepsilon_{\alpha \beta}^{f V}$ in units of $G_{F}$. Then the effective charge in Eq. (2.2) can be written as

$$
Q_{\alpha}^{2}=\left[Z\left(g_{p}^{V}+2 \varepsilon_{\alpha \alpha}^{u V}+\varepsilon_{\alpha \alpha}^{d V}\right)+N\left(g_{n}^{V}+\varepsilon_{\alpha \alpha}^{u V}+2 \varepsilon_{\alpha \alpha}^{d V}\right)\right]^{2}+\sum_{\beta \neq \alpha}\left[Z\left(2 \varepsilon_{\alpha \beta}^{u V}+\varepsilon_{\alpha \beta}^{d V}\right)+N\left(\varepsilon_{\alpha \beta}^{u V}+2 \varepsilon_{\alpha \beta}^{d V}\right)\right]^{2} .
$$

For simplicity, we consider four cases with only two nonzero NSI parameters: (a) $\varepsilon_{e e}^{u V} \neq 0, \varepsilon_{e e}^{d V} \neq 0$; (b) $\varepsilon_{\mu \mu}^{u V} \neq 0, \varepsilon_{\mu \mu}^{d V} \neq 0$; (c) $\varepsilon_{e e}^{d V} \neq 0, \varepsilon_{\mu \mu}^{d V} \neq 0$; and (d) $\varepsilon_{e e}^{d V} \neq 0, \varepsilon_{e \tau}^{d V} \neq 0$. We find that due to degeneracies between different combinations of NSI parameters, especially the cancellation between the NSI coupling to up and down quarks, large values of NSI parameters are allowed. However, the effective NSI parameters that affect neutrino oscillation experiments on Earth depend on the sum of the up-type and down-type NSI parameters. Therefore, the COHERENT data can still place meaningful constraints on the effective NSI parameters in Earth matter. As an example, we find 
that the effective NSI parameters lie in the ranges $-0.95 \leq \varepsilon_{e e} \leq 1.95$ and $-0.66 \leq \varepsilon_{\mu \mu} \leq 1.57$ at $90 \%$ CL if only these two effective NSI parameters are nonzero.

\section{Conclusion}

The coherent elastic neutrino-nucleus scattering has small moment transfer and large cross section, hence it is desirable to use CEVNS to study nonstandard neutrino interactions induced by a light mediator. We analyzed the spectrum of the COHERENT data and used it constrain NSI induced by both a light and heavy mediator. We find that for a very light mediator, the COHERENT data only constrain the mediator coupling, and it cannot be directly applied to matter NSI because the NSI matter effect in neutrino propagation depends on $\frac{g^{2}}{M_{Z^{\prime}}^{2}}$. We also find that the COHERENT constraints are weakened by degeneracies between different combinations of NSI parameters for a heavier mediator. However, meaningful constraints can still be placed on the effective NSI parameters in Earth matter since they are not affected by the cancellation between the NSI couplings to the up and down quarks.

JL and DM acknowledge support from the U.S. DOE under Grant No. DE-SC0010504

\section{References}

[1] Y. Farzan and M. Tortola, arXiv:1710.09360 [hep-ph].

[2] J. Liao, D. Marfatia and K. Whisnant, Phys. Lett. B 767, 350 (2017) [arXiv:1609.01786 [hep-ph]].

[3] J. Liao, D. Marfatia and K. Whisnant, Phys. Lett. B 771, 247 (2017) [arXiv:1704.04711 [hep-ph]].

[4] J. Liao, D. Marfatia and K. Whisnant, Phys. Rev. D 93, no. 9, 093016 (2016) [arXiv:1601.00927].

[5] J. Liao, D. Marfatia and K. Whisnant, JHEP 1701, 071 (2017) [arXiv:1612.01443 [hep-ph]].

[6] P. Coloma, P. B. Denton, M. C. Gonzalez-Garcia, M. Maltoni and T. Schwetz, JHEP 1704, 116 (2017) [arXiv:1701.04828 [hep-ph]].

[7] K. Scholberg, Phys. Rev. D 73, 033005 (2006) [hep-ex/0511042].

[8] D. Akimov et al. [COHERENT Collaboration], Science 357, no. 6356, 1123 (2017) [arXiv:1708.01294 [nucl-ex]].

[9] J. I. Collar, N. E. Fields, M. Hai, T. W. Hossbach, J. L. Orrell, C. T. Overman, G. Perumpilly and B. Scholz, Nucl. Instrum. Meth. A 773, 56 (2015) [arXiv:1407.7524 [physics.ins-det]].

[10] S. R. Klein and J. Nystrand, Phys. Rev. Lett. 84, 2330 (2000) [hep-ph/9909237].

[11] J. Liao and D. Marfatia, Phys. Lett. B 775, 54 (2017) [arXiv:1708.04255 [hep-ph]].

[12] F. Jegerlehner and A. Nyffeler, Phys. Rept. 477, 1 (2009).

[13] Y. Farzan, Phys. Lett. B 748 (2015) 311 [arXiv:1505.06906 [hep-ph]]. 